\title{
Supporting Information \\ In-Situ Anchoring Polymetallic Phosphide \\ Nanoparticles within Porous Prussian Blue \\ Analogue Nanocages for Boosting Oxygen \\ Evolution Catalysis
}

Guangxun Zhang, ${ }^{\dagger}$ Yanle Li,, Xiao Xiao, ${ }^{\dagger}$ Yang Shan,$^{\dagger}$ Yang Bai, ${ }^{\dagger}$ Huai-Guo Xue, ${ }^{\dagger}$

Huan Pang, ${ }^{* \dagger}$ Ziqi Tian, ${ }^{*,+}$ and Qiang $X u^{*,+,,, \uparrow}$

$\uparrow$ School of Chemistry and Chemical Engineering, Yangzhou University, Yangzhou, 225009, Jiangsu, P. R. China.

\$ Ningbo Institute of Materials Technology and Engineering, Chinese Academy of Sciences, Ningbo 315201, Zhejiang, P. R. China.

$\S$ Department of Materials Science and Engineering, Southern University of Science and Technology (SUSTech), Shenzhen 518055, P. R. China.

I Institute for Integrated Cell-Material Sciences (iCeMS), Kyoto University, Yoshida, Sakyo-ku, Kyoto 606-8501, Japan.

*Email: huanpangchem@hotmail.com; panghuan@yzu.edu.cn (H. Pang); tianziqi@nimte.ac.cn (Z.Tian); qxuchem@yzu.edu.cn; xuq@sustech.edu.cn; xu.qiang@icems.kyoto-u.ac.jp (Q. Xu) 


\section{Materials and methods}

\section{Chemicals and reagents:}

Nickel(II) nitrate hexahydrate $\left(\mathrm{Ni}\left(\mathrm{NO}_{3}\right)_{2} \cdot 6 \mathrm{H}_{2} \mathrm{O}, \geq 99 \%\right)$, Cobalt (II) nitrate hexahydrate $\left(\mathrm{Co}\left(\mathrm{NO}_{3}\right)_{2} \cdot 6 \mathrm{H}_{2} \mathrm{O}, \geq 98.0 \%\right)$, Trisodium citrate dihydrate $\left(\mathrm{Na}_{3} \mathrm{C}_{6} \mathrm{H}_{5} \mathrm{O}_{7} \cdot 2 \mathrm{H}_{2} \mathrm{O}, \geq 99.0 \%\right)$, Potassium hexacyanoferrate (III) $\left(\mathrm{K}_{3}\left[\mathrm{Fe}(\mathrm{CN})_{6}\right], \geq\right.$ $99.5 \%)$, and Sodium hypophosphite $\left(\mathrm{NaH}_{2} \mathrm{PO}_{2},>88.0 \%\right)$ were used as analyticalgrade reagents without further purification.

\section{Materials synthesis}

Synthesis of NiCoFe-PBA nanocubes: In a typical procedure, $0.1454 \mathrm{~g}$ of Nickel(II) nitrate hexahydrate, $0.4365 \mathrm{~g}$ of cobalt nitrate hexahydrate, and $0.6617 \mathrm{~g}$ trisodium citrate dihydrate were dissolved in $50 \mathrm{~mL}$ of deionized water to generate clear solution A. Then, $0.3293 \mathrm{~g}$ potassium hexacyanoferrate(III) was dissolved in $50 \mathrm{~mL}$ of deionized water to form solution B. Then, solution B was added into solution A under magnetic stirring at room temperature. After continuous stirring for $10 \mathrm{~min}$, the obtained mixed solution was aged for another $24 \mathrm{~h}$ at room temperature. The precipitate was washed via several rinsing-centrifugation cycles with deionized water and ethanol and then dried at $50{ }^{\circ} \mathrm{C}$ overnight. The obtained product was designated NiCoFe-PBA.

Synthesis of NiFe-PBA nanocubes: In a typical procedure, $0.5816 \mathrm{~g}$ of Nickel(II) nitrate hexahydrate and $0.6617 \mathrm{~g}$ trisodium citrate dihydrate were dissolved in $50 \mathrm{~mL}$ of deionized water to generate clear solution A. Then, $0.3293 \mathrm{~g}$ potassium hexacyanoferrate(III) was dissolved in $50 \mathrm{~mL}$ of deionized water to form solution B. Then, solution B was added into solution A under magnetic stirring at room temperature. 
After continuous stirring for $10 \mathrm{~min}$, the obtained mixed solution was aged for another $24 \mathrm{~h}$ at room temperature. The precipitate was washed via several rinsing-centrifugation cycles with deionized water and ethanol and then dried at $50{ }^{\circ} \mathrm{C}$ overnight. The obtained product was designated NiFe-PBA.

Synthesis of CoFe-PBA nanocubes: In a typical procedure, $0.5821 \mathrm{~g}$ of cobalt nitrate hexahydrate, and $0.6617 \mathrm{~g}$ trisodium citrate dihydrate were dissolved in $50 \mathrm{~mL}$ of deionized water to generate clear solution A. Then, $0.3293 \mathrm{~g}$ potassium hexacyanoferrate(III) was dissolved in $50 \mathrm{~mL}$ of deionized water to form solution $\mathrm{B}$. Then, solution B was added into solution A under magnetic stirring at room temperature. After continuous stirring for $10 \mathrm{~min}$, the obtained mixed solution was aged for another $24 \mathrm{~h}$ at room temperature. The precipitate was washed via several rinsing-centrifugation cycles with deionized water and ethanol and then dried at $50{ }^{\circ} \mathrm{C}$ overnight. The obtained product was designated CoFe-PBA.

\section{Synthesis of NiCoFe-P-NP@NiCoFe-PBA porous nanocages and NiFe-P-}

NP@NiFe-PBA sample: $50 \mathrm{mg}$ NiCoFe-PBA nanocubes and $300 \mathrm{mg} \mathrm{NaH} \mathrm{PO}_{2}$ were placed at two separate positions in a porcelain boat with $\mathrm{NaH}_{2} \mathrm{PO}_{2}$ powder at the upstream side of the tube furnace. The phosphidation process was carried out under a nitrogen atmosphere and the tube furnace was heated to $300{ }^{\circ} \mathrm{C}$ at a rate of $1{ }^{\circ} \mathrm{C} \mathrm{min}^{-1}$ and maintained this temperature for $150 \mathrm{~min}$. The NiCoFe-P-NP@NiCoFe-PBA porous nanocages were subsequently collected after the sample cooled to room temperature. Using NiFe-PBA as the precursor, we synthesized the NiFe-P-NP@NiFe-PBA sample via the same thermal phosphidation procedure. 
Synthesis of CoFe-P-NP@CoFe-PBA sample: $50 \mathrm{mg}$ CoFe-PBA nanocubes and 300 mg $\mathrm{NaH}_{2} \mathrm{PO}_{2}$ were placed at two separate positions in a porcelain boat with $\mathrm{NaH}_{2} \mathrm{PO}_{2}$ powder at the upstream side of the tube furnace. The phosphidation process was carried out under a nitrogen atmosphere and the tube furnace was heated to $350{ }^{\circ} \mathrm{C}$ at a rate of $2{ }^{\circ} \mathrm{C} \mathrm{min}-1$ and maintained this temperature for $3 \mathrm{~h}$. The CoFe-P-NP@CoFe-PBA sample were subsequently collected after the sample cooled to room temperature.

Synthesis of NiCoFe-P sample: $40 \mathrm{mg}$ NiCoFe-PBA nanocubes and $400 \mathrm{mg}$ $\mathrm{NaH}_{2} \mathrm{PO}_{2}$ were placed at two separate positions in a porcelain boat with $\mathrm{NaH}_{2} \mathrm{PO}_{2}$ powder at the upstream side of the tube furnace. The phosphidation process was carried out under a nitrogen atmosphere and the tube furnace was heated to $350{ }^{\circ} \mathrm{C}$ at a rate of $2{ }^{\circ} \mathrm{C} \min ^{-1}$ and maintained this temperature for $4 \mathrm{~h}$. The NiCoFe-P sample were subsequently collected after the sample cooled to room temperature.

\section{Material characterization}

Characterization of TMP, PBA and TMP@PBA architectures: The products were tested by X-ray diffraction (XRD) on a Bruker D8 Advanced X-ray Diffractometer $(\mathrm{Cu}-\mathrm{K} \alpha$ radiation: $\lambda=0.15406 \mathrm{~nm}$ ) for the phase analysis. Fourier Transform Infrared Spectrometer (FT-IR) measurement was investigated on a TENSOR27. Scanning electron microscope (SEM, Zeiss-Supra55) was used for observing the morphology of the samples at an acceleration voltage of $5.0 \mathrm{kV}$. High-resolution transmission electron microscopy (HRTEM) images, SAED images, and energy dispersive X-ray spectroscopy mapping were captured on a Tecnai G2 F30 transmission electron microscopy at an acceleration voltage of $300 \mathrm{kV}$. X-ray photoelectron spectroscopy 
(XPS) was carried out on a Thermo Scientific ESCALAB 250 apparatus. EPR spectra were recorded on a Bruker A300 electron paramagnetic resonance spectrometer at room temperature.

\section{Electrochemical measurements}

Electrochemical performance experiments were carried out using a CHI 760e Electrochemical Workstation $(\mathrm{CH}$ Instruments, Shanghai Chenhua Instrument Corporation, China) in a (non-noble) three-electrode system. The electrochemical water oxidation experiments were carried out on an electrochemical working station. The three-electrode system was implemented for all the electrochemical measurements by utilizing glassy carbon electrode (GCE) (diameter: $3.0 \mathrm{~mm}$ ) as working electrode, graphite rod as counter electrode, and $\mathrm{Hg}-\mathrm{HgO}$ electrode as reference electrode in 1.0 $\mathrm{M} \mathrm{KOH}$ electrolyte. $10 \mathrm{mg}$ of catalyst was dispersed in Nafion solution with a concentration of approximate $10.0 \mathrm{mg} \mathrm{mL}^{-1}$ followed by ultrasonication for $0.5 \mathrm{~h}$. After that, the working electrode coated with $5 \mu \mathrm{L}$ of the above catalyst, and dried naturally. Prior to the electrochemical test, we first passed half an hour of $\mathrm{N}_{2}$ gas in the $1.0 \mathrm{M}$ $\mathrm{KOH}$ to ensure the $\mathrm{O}_{2} / \mathrm{H}_{2} \mathrm{O}$ equilibrium at $1.23 \mathrm{~V}$ vs. RHE.

\section{Theoretical Calculations}

The Perdew-Burke-Ernzerhof (PBE) functional with the projector augmented wave (PAW) method was applied to describe the interaction between ions and electrons. The DFT-D2 correction was utilized to consider the van der Waals interactions. Both experimental and theoretical investigations indicated $\mathrm{CoP}(111)$ surface to be active in 
water splitting. ${ }^{1-3}$ Thus the CoP slab model was built from three layers of $(2 \times 2)$ CoP(111) facet with a $15 \AA$ vacuum slab. The complex surface is constructed by placing CoP cluster on PBA (001) surface. The cluster was cut from $\mathrm{CoP}(111)$ model that contains $20 \mathrm{Co}$ and $20 \mathrm{P}$ atoms. Only the top layer in $\mathrm{CoP}(111)$ model, the $(\mathrm{CoP})_{20}$ cluster within the complex and adsorbed species were allowed to relax during the optimization. The cutoff energy of $400 \mathrm{eV}$ for the plane-wave basis set was used. The convergence threshold for structural optimization was set to be $0.025 \mathrm{eV} \AA^{-1}$ in force. The k-points meshing for Brillioun zone was set as $5 \times 5 \times 1$ for $\mathrm{CoP}(111)$ and Gama point for the complex.

The four-electron process was considered to study the OER mechanism, including the following four elementary steps:

$\mathrm{H}_{2} \mathrm{O}+* \rightarrow * \mathrm{OH}+\mathrm{H}^{+}+\mathrm{e}^{-}$

$* \mathrm{OH} \rightarrow * \mathrm{O}+\mathrm{H}^{+}+\mathrm{e}^{-}$

$\mathrm{H}_{2} \mathrm{O}+* \mathrm{O} \rightarrow * \mathrm{OOH}+\mathrm{H}^{+}+\mathrm{e}^{-}$

${ }^{*} \mathrm{OOH} \rightarrow{ }^{*}+\mathrm{O}_{2}+\mathrm{H}^{+}+\mathrm{e}^{-}$

where asterisk $(*)$ denotes an adsorption site on the surface. The free energy change of each step could be calculated with the following equations:

$$
\begin{aligned}
& \Delta \mathrm{G}_{1}=\mathrm{E}(* \mathrm{OH})-\mathrm{E}(*)-\mathrm{E}_{\mathrm{H} 2 \mathrm{O}}+1 / 2 \mathrm{E}_{\mathrm{H} 2}+(\Delta \mathrm{ZPE}-\mathrm{T} \Delta \mathrm{S}) \\
& \Delta \mathrm{G}_{2}=\mathrm{E}(* \mathrm{O})-\mathrm{E}(* \mathrm{OH})+1 / 2 \mathrm{E}_{\mathrm{H} 2}+(\Delta \mathrm{ZPE}-\mathrm{T} \Delta \mathrm{S}) \\
& \Delta \mathrm{G}_{3}=\mathrm{E}(* \mathrm{OOH})-\mathrm{E}(* \mathrm{O})-\mathrm{E}_{\mathrm{H} 2 \mathrm{O}}+1 / 2 \mathrm{E}_{\mathrm{H} 2}+(\Delta \mathrm{ZPE}-\mathrm{T} \Delta \mathrm{S}) \\
& \Delta \mathrm{G}_{4}=\mathrm{E}(*)-\mathrm{E}(* \mathrm{OOH})+\mathrm{E}_{\mathrm{O} 2}+1 / 2 \mathrm{E}_{\mathrm{H} 2}+(\Delta \mathrm{ZPE}-\mathrm{T} \Delta \mathrm{S})
\end{aligned}
$$

where $\mathrm{E}\left({ }^{*}\right), \mathrm{E}\left({ }^{*} \mathrm{OH}\right), \mathrm{E}\left({ }^{*} \mathrm{O}\right)$ and $\mathrm{E}\left({ }^{*} \mathrm{OOH}\right)$ are the computed energies of the surface and 
the adsorbed $* \mathrm{OH}, * \mathrm{O}$ and $* \mathrm{OOH}$ species, respectively. The zero point energy and the entropy included in $\Delta \mathrm{G}_{1} \sim \Delta \mathrm{G}_{4}$ are taken from the reference. ${ }^{4}$ The Co cations locate on the $24 \mathrm{~d}$ (face-centered) sites, which is the Wyckoff notations in per cubic unit cell, and similar to $\mathrm{Na}^{+}$in the NiFeCo prussian blue analogs. ${ }^{5}$
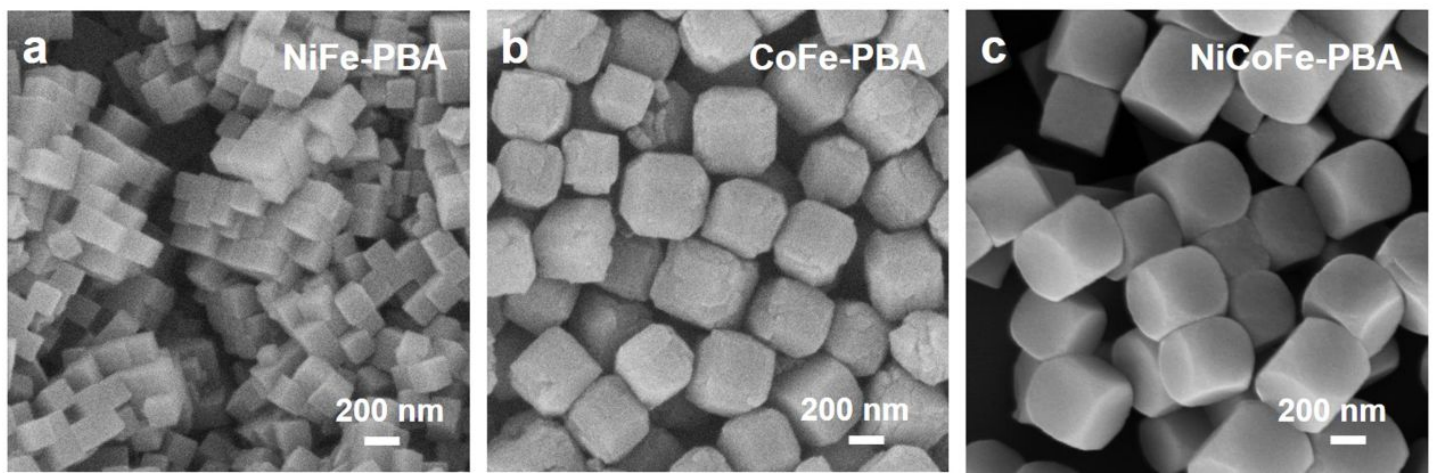

Figure S1. SEM images of the three PBA precursors.
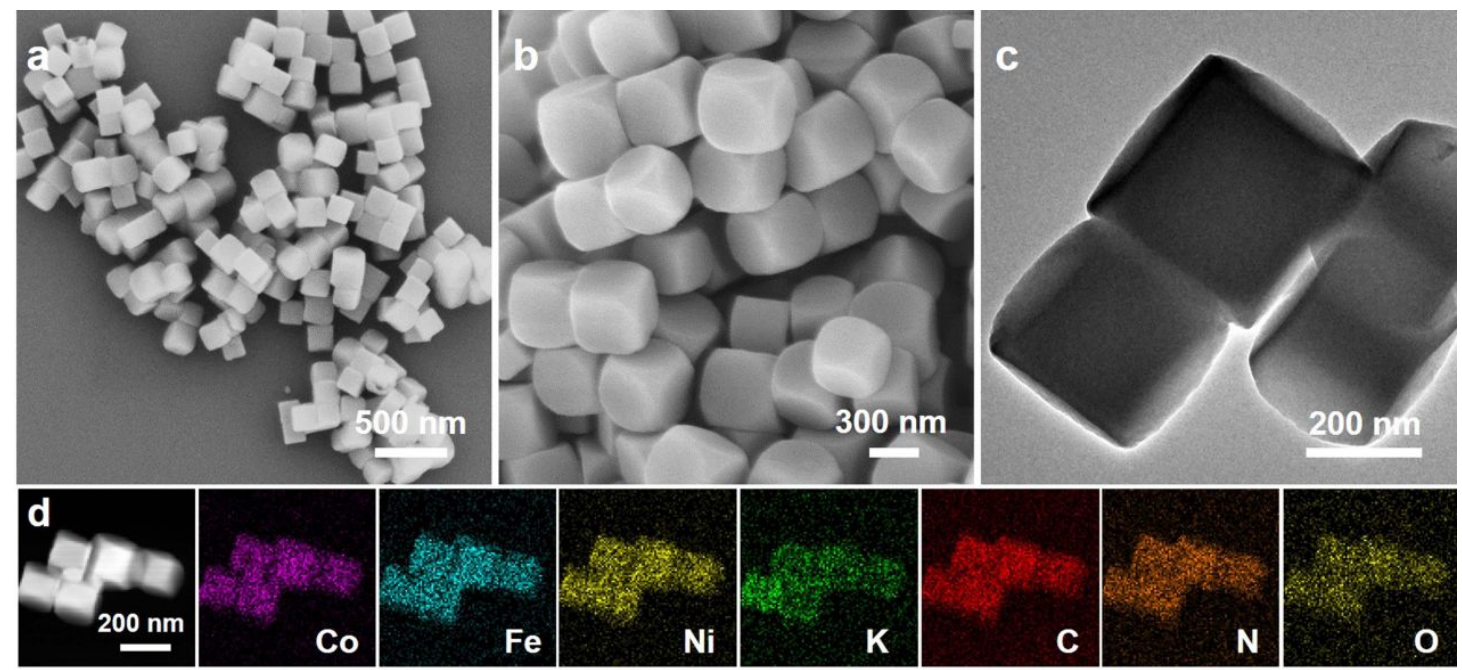

Figure S2. (a-b) SEM images and (c) TEM image of the NiCoFe-PBA precursors. (d) EDS elemental mapping images of the NiCoFe-PBA precursors. 


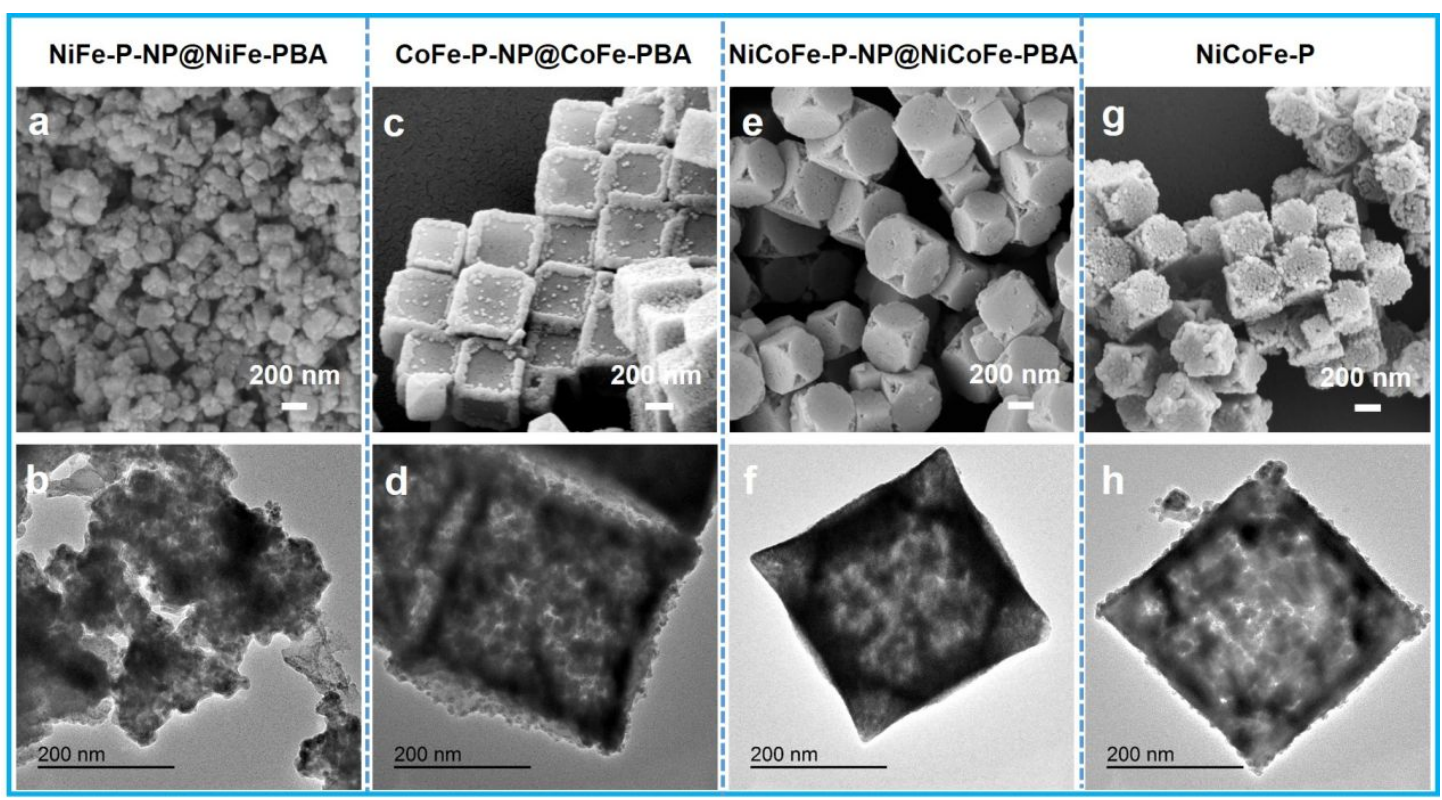

Figure S3. $(a, c, e, g)$ SEM images and $(b, d, f, h)$ TEM images of the four phosphidation products.

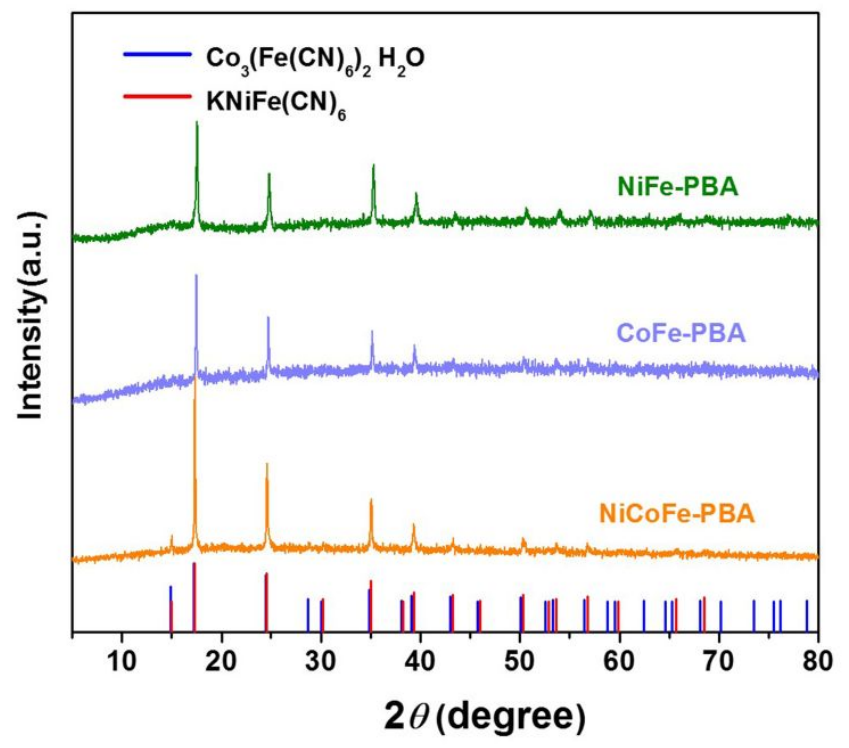

Figure S4. XRD patterns of the three PBA precursors. 


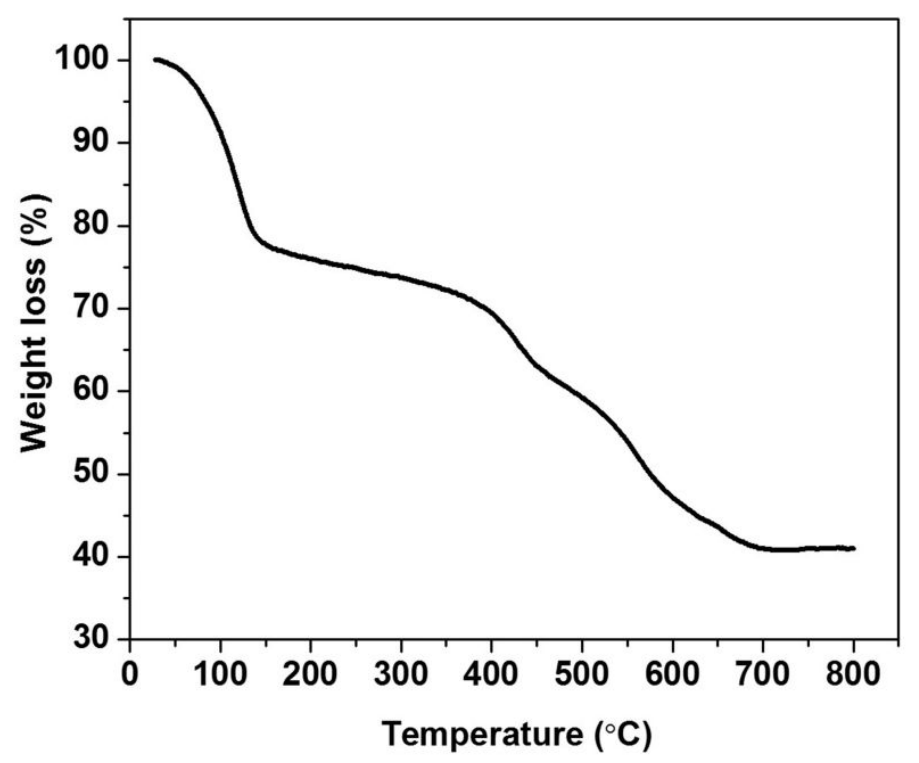

Figure S5. Thermogravimetric (TG) curve of the NiCoFe-PBA precursor measured under a $\mathrm{N}_{2}$ atmosphere. TG analyses of the $\mathrm{NiCoFe}-\mathrm{PBA}$ precursor can provide some information about the formation mechanism of the NiCoFe-P-NP@NiCoFe-PBA porous nanocage. The NiCoFe-PBA nanocube suffers from a large mass loss below 145 ${ }^{\circ} \mathrm{C}$, most probably because of $\mathrm{H}_{2} \mathrm{O}$ loss. As the temperature was increased from 145 to $300{ }^{\circ} \mathrm{C}$, the weight reduces gradually, which could be attributed to the removal of the coordinating $\mathrm{H}_{2} \mathrm{O}$ in the PBA precursor. The removal of $\mathrm{H}_{2} \mathrm{O}$ probably led to the formation of a large number of pore structures. The mass loss from 300 to $500{ }^{\circ} \mathrm{C}$ could be ascribed to the stepwise decomposition of the cyano group in NiCoFe-PBA. Hence, the transition metal ions are partially reduced via liberation of the cyano group to the metallic state in the low-temperature phosphidation process, and they react with the hydrogen phosphide released from $\mathrm{NaH}_{2} \mathrm{PO}_{2}$ to produce heterogeneous polymetallic phosphides. 


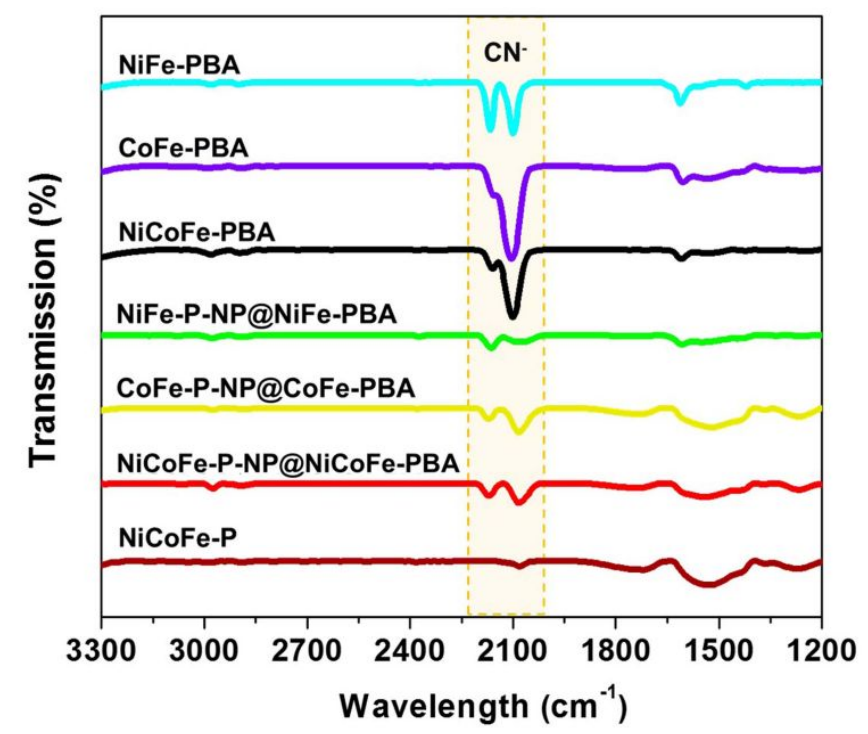

Figure S6. FTIR of the different PBA precursors and obtained different TMP@PBA and NiCoFe-P nanocages. The peaks observed in $1605 \mathrm{~cm}^{-1}$ for NiCoFe-PNP@NiCoFe-PBA and the control samples are attributed to the bending vibration of $\mathrm{H}_{2} \mathrm{O}$ molecules. The characteristic adsorption bands located at the region of 2010-2210 $\mathrm{cm}^{-1}$ correspond to the stretching vibration of the cyano group. The three types of PBA precursors have a strong cyano absorption band before the phosphidation treatment. The FTIR results of the NiCoFe-P-NP@NiCoFe-PBA hybrids reveal coexistence of the ternary metallic phosphide nanoparticles and the PBA porous nanocages.

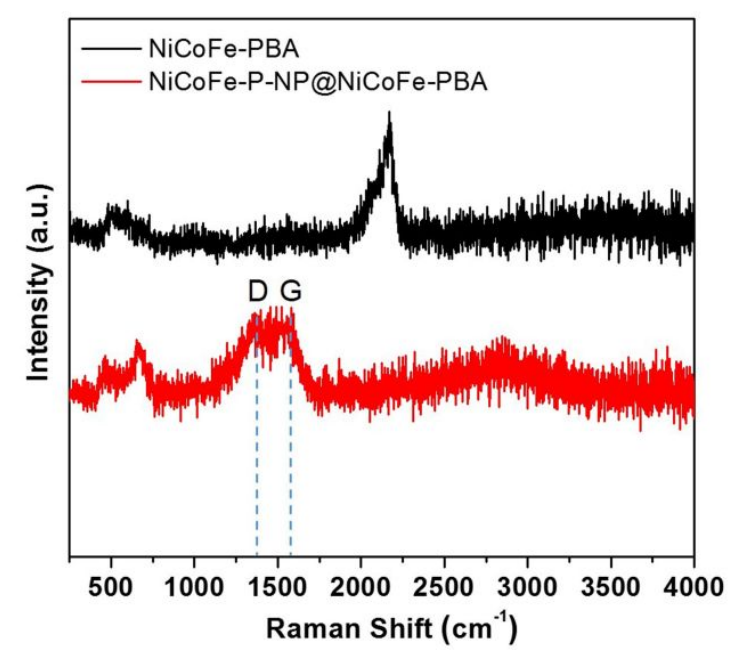

Figure S7. Raman of the NiCoFe-PBA precursor and porous NiCoFe-P-NP@NiCoFePBA nanocages. The Raman measurement on the NiCoFe-PBA precursor displays an 
obvious peak at $2165 \mathrm{~cm}^{-1}$, corresponding to the vibration of the $\mathrm{CN}$ cyano group in the mixture of Fe $\mathrm{Fe}^{\mathrm{II}}-\mathrm{CN}-\mathrm{Ni}{ }^{\mathrm{III}}$. The peak at $2165 \mathrm{~cm}^{-1}$ weakens for the NiCoFe-P$\mathrm{NP} @ \mathrm{NiCoFe}-\mathrm{PBA}$ sample, meaning that a number of NiCoFe-PBA species converted to metal phosphides after the phosphidation reaction. The $\mathrm{D}$ band $\left(\mathrm{I}_{\mathrm{D}}\right)$ corresponds to the induced disorder. The $\mathrm{G}$ band $\left(\mathrm{I}_{\mathrm{G}}\right)$ corresponds to the intrinsic feature of $\mathrm{sp}^{2}$ carbon. The band intensity ratio $\left(\mathrm{I}_{\mathrm{D}} / \mathrm{I}_{\mathrm{G}}\right)$ of the NiCoFe-P-NP@NiCoFe-PBA nanocages was 1.06, suggesting the formation of lattice defects during the phosphidation process. Furthermore, the catalytic activity of metal phosphide is related to its surface properties and electronic structures, and could be improved by increasing the quantity of exposed active sites and optimizing its electronic structures by rationally tuning the morphology, surface area or defects. ${ }^{11}$

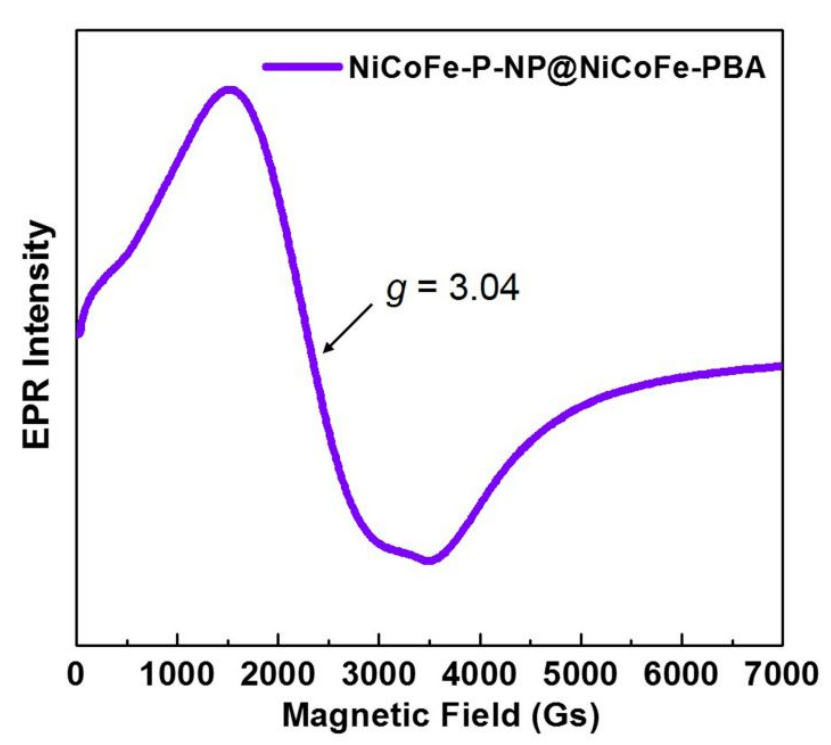

Figure S8. Electron paramagnetic resonance (EPR) spectra of the porous NiCoFe-PNP@NiCoFe-PBA nanocages at room temperature. 

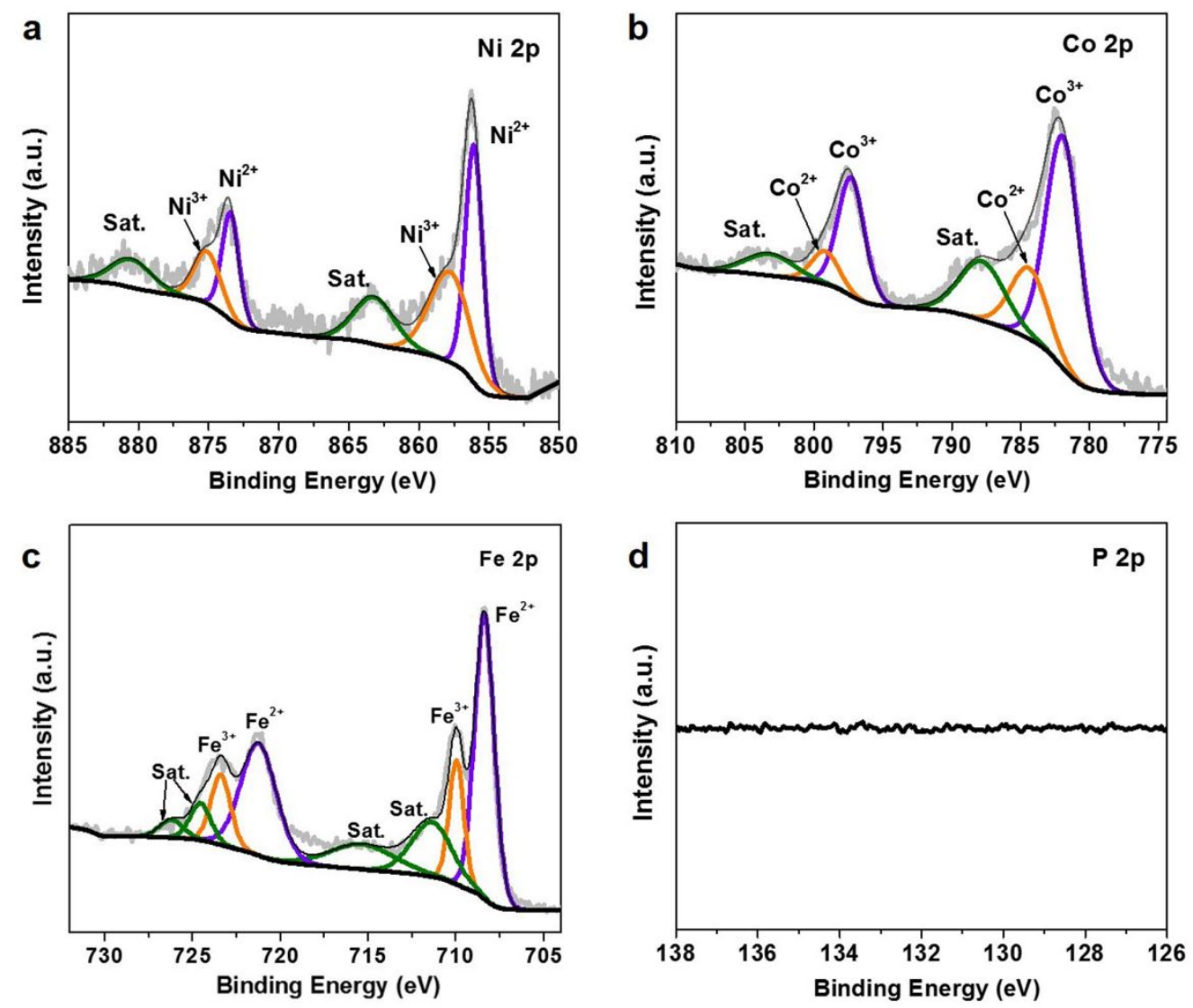

Figure S9. High-resolution XPS spectra of (a) Ni 2p, (b) Co 2p, (c) Fe 2p, and (d) P 2p of NiCoFe-PBA precursor. (a) The $\mathrm{Ni} 2 \mathrm{p}_{3 / 2}$ spectrum of NiCoFe-PBA can be deconvoluted into $\mathrm{Ni}^{3+}$ in $\mathrm{NiO}_{\mathrm{x}},(857.81 \mathrm{eV}), \mathrm{Ni}^{2+}$ in $\mathrm{NiCoFe}-\mathrm{PBA}(856.08 \mathrm{eV})$ and satellite peaks $(863.18 \mathrm{eV})$. (b) For the Co $2 \mathrm{p}_{3 / 2}$ spectrum of NiCoFe-PBA precursor, the peaks at 784.39, 781.94, and 787.79 eV are well assigned to $\mathrm{Co}^{2+}$ in $\mathrm{NiCoFe}-\mathrm{PBA}$, $\mathrm{Co}^{3+}$ in oxidized Co species, and satellite peaks, respectively. (c) Three Fe types in the NiCoFe-PBA precursor, including $\mathrm{Fe}^{3+} / \mathrm{Fe}^{2+}$ in NiCoFe-PBA $(709.97 \mathrm{eV} / 708.38 \mathrm{eV})$ and satellite peaks (711.37 eV and $715.35 \mathrm{eV}$ ), could be distinguished from the Fe $2 \mathrm{p}_{3 / 2}$ spectrum. (d) There is no characteristic peak of $\mathrm{P}$ in the NiCoFe-PBA precursor. 


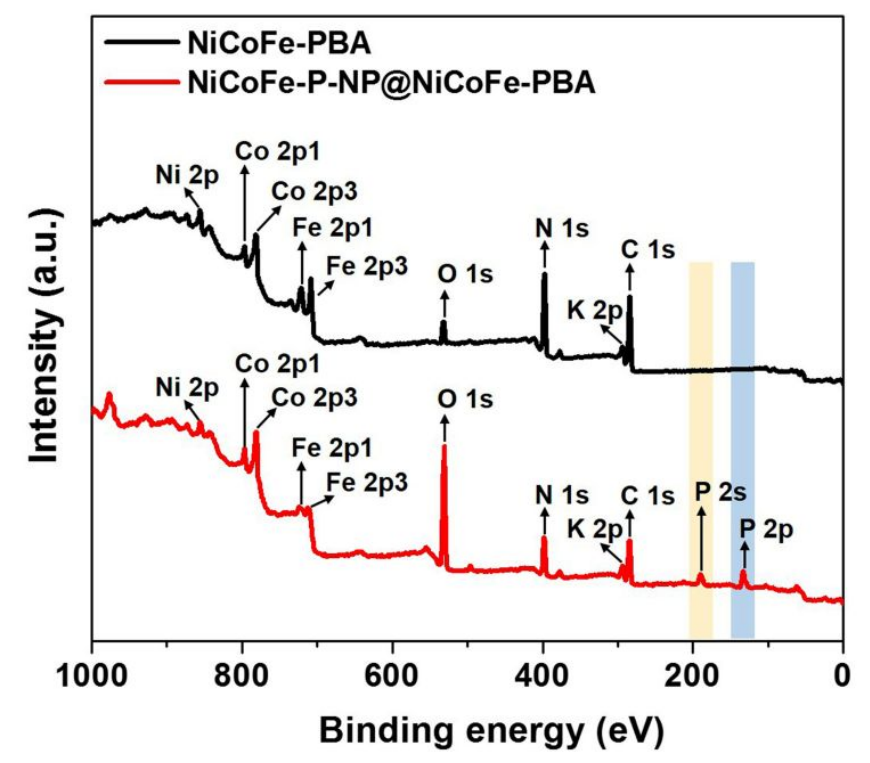

Figure S10. XPS spectra of the NiCoFe-PBA nanocubes and the obtained porous NiCoFe-P-NP@NiCoFe-PBA nanocages. The overall XPS spectrum of the NiCoFeP@NiCoFe-PBA nanocages manifests the coexistence of P, Ni, Co, Fe, K, C, $\mathrm{N}$, and $\mathrm{O}$ elements, also matching with the EDX elemental mapping analysis in Figure $2 \mathrm{j}-\mathrm{q}$.

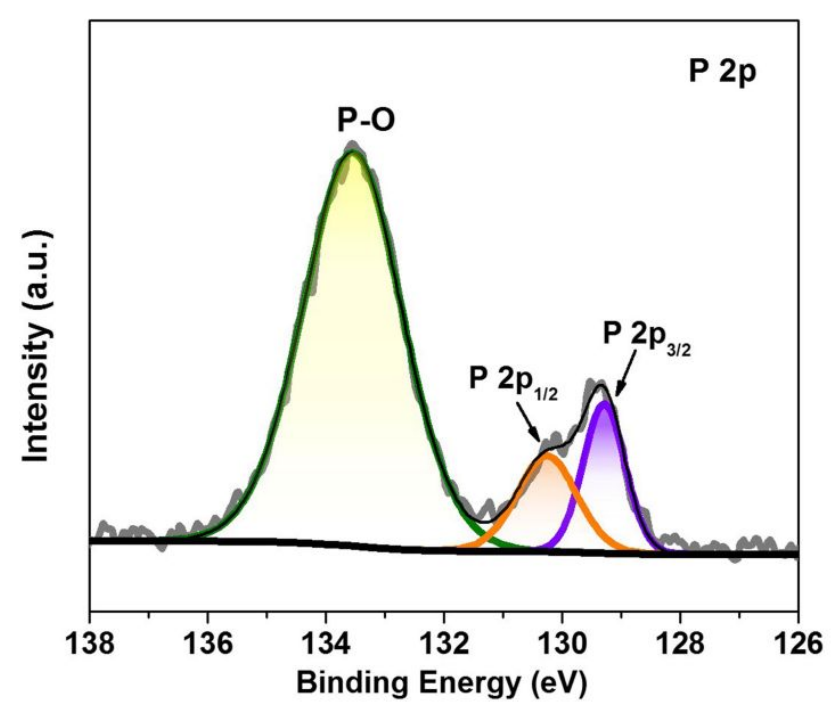

Figure S11. High-resolution XPS spectra of P 2p of NiCoFe-P-NP@NiCoFe-PBA nanocages. Compared with the $\mathrm{BE}$ of $130 \mathrm{eV}$ in elemental $\mathrm{P}$, the $\mathrm{BE}$ of $\mathrm{P} 2 \mathrm{p}_{3 / 2}$ has a negative shift, indicating that P in the NiCoFe-P-NP@NiCoFe-PBA hybrids have a partially negative charge ( $\delta^{-}$in $\mathrm{CoP}$ and $\mathrm{FeP}, 2 \delta^{-}$in $\left.\mathrm{Ni}_{2} \mathrm{P}\right)$. 

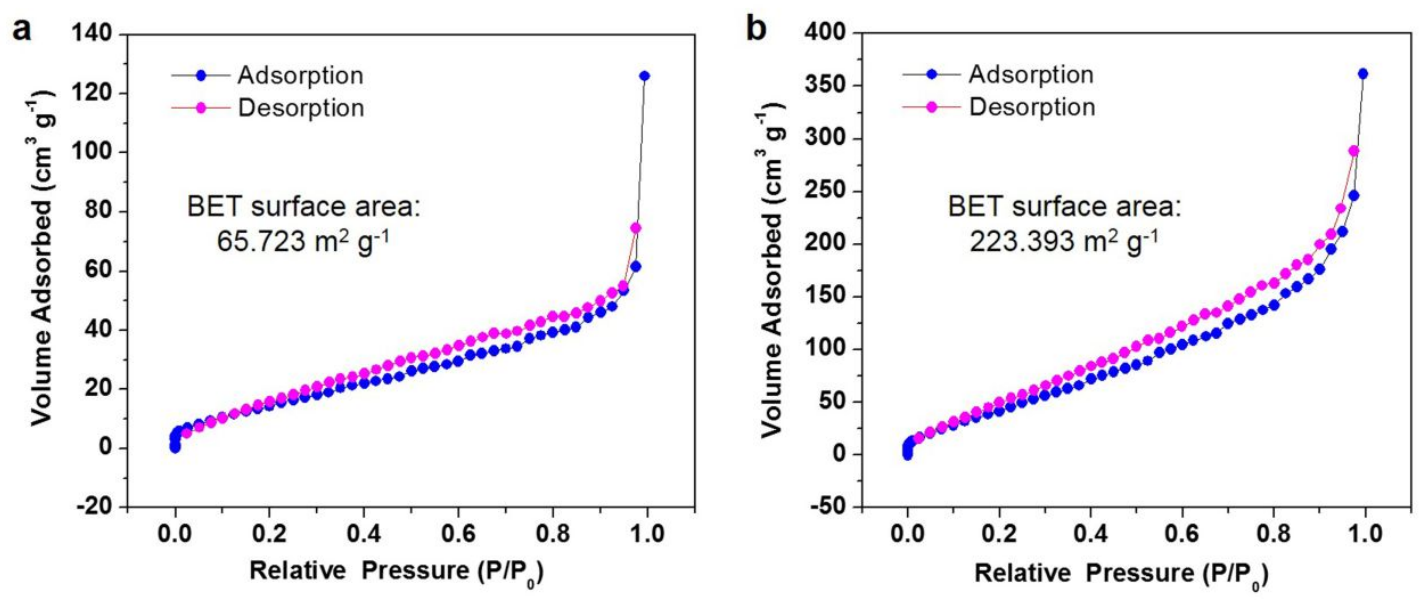

Figure S12. The $\mathrm{N}_{2}$ sorption isotherms of (a) the NiCoFe-PBA precursor and (b) NiCoFe-P-NP@NiCoFe-PBA nanocage. The results indicate that the specific surface area of obtained NiCoFe-P-NP@NiCoFe-PBA nanocages have an obvious improvement after the phosphidation, which is beneficial to the oxygen evolution process.

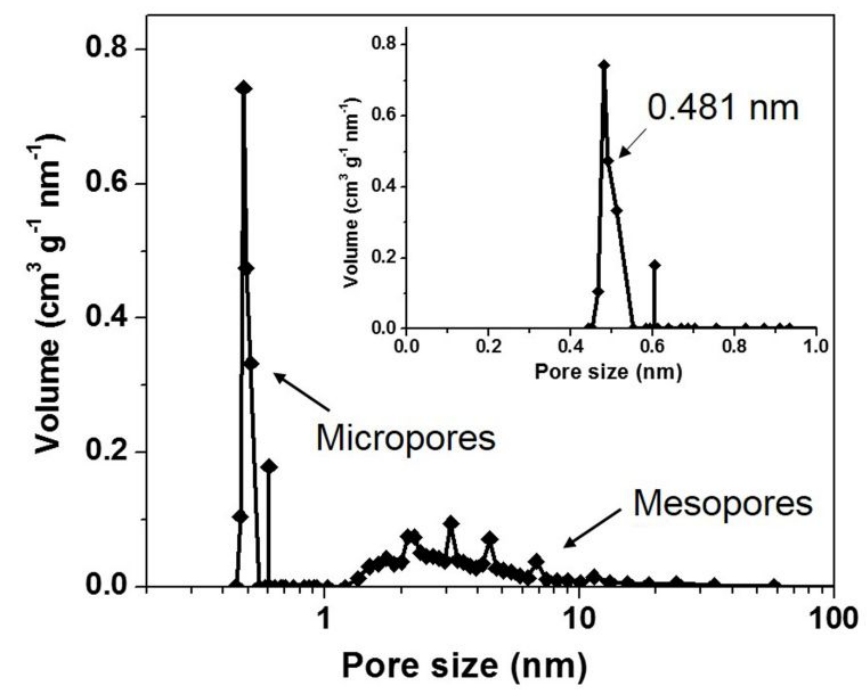

Figure S13. Pore diameter distribution of the porous NiCoFe-P-NP@NiCoFe-PBA nanocages. The pore size distribution of the NiCoFe-P-NP@NiCoFe-PBA porous nanocage was obtained using the Barrett-Joyner-Halenda (BJH) method. 
Table S1. Comparison of OER activity in alkaline electrolyte (1.0 M KOH) for porous NiCoFe-P-NP@NiCoFe-PBA nanocage with other recently published highly active OER electrocatalysts.

\begin{tabular}{|c|c|c|c|c|c|}
\hline Catalysts & $\begin{array}{l}\text { Current } \\
\text { density } \\
(j)\end{array}$ & $\begin{array}{l}\text { Overpotential } \\
\quad(y) \text { at }(j)\end{array}$ & Tafel slope & Electrolyte & Ref. \\
\hline $\begin{array}{c}\text { This work } \\
\text { (porous NiCoFe-P- } \\
\text { NP@NiCoFe-PBA } \\
\text { nanocage) }\end{array}$ & $10 \mathrm{~mA} \mathrm{~cm}^{-2}$ & $223 \mathrm{mV}$ & $78 \mathrm{mV} \mathrm{dec}^{-1}$ & $1.0 \mathrm{M} \mathrm{KOH}$ & \\
\hline Black phosphorus $/ \mathrm{Co}_{2} \mathrm{P}$ & $10 \mathrm{~mA} \mathrm{~cm}^{-2}$ & $380 \mathrm{mV}$ & $78 \mathrm{mV} \mathrm{dec}^{-1}$ & $1.0 \mathrm{M} \mathrm{KOH}$ & 6 \\
\hline CoP nanoframes & $10 \mathrm{~mA} \mathrm{~cm}^{-2}$ & $323 \mathrm{mV}$ & $49.6 \mathrm{mV} \mathrm{dec}-1$ & $1.0 \mathrm{M} \mathrm{KOH}$ & 7 \\
\hline Fe-Co-P alloy & $10 \mathrm{~mA} \mathrm{~cm}^{-2}$ & $252 \mathrm{mV}$ & $33 \mathrm{mV} \mathrm{dec}^{-1}$ & $1.0 \mathrm{M} \mathrm{KOH}$ & 8 \\
\hline $\mathrm{Ni}_{0.6} \mathrm{Co}_{1.4} \mathrm{P}$ nanocages & $10 \mathrm{~mA} \mathrm{~cm}^{-2}$ & $300 \mathrm{mV}$ & $80 \mathrm{mV} \mathrm{dec}^{-1}$ & $1.0 \mathrm{M} \mathrm{KOH}$ & 9 \\
\hline $\mathrm{FeP}_{2}$ & $10 \mathrm{~mA} \mathrm{~cm}^{-2}$ & $240 \mathrm{mV}$ & $56 \mathrm{mV} \mathrm{dec}^{-1}$ & $1.0 \mathrm{M} \mathrm{KOH}$ & 10 \\
\hline NiCoP-nanosheets & $10 \mathrm{~mA} \mathrm{~cm}^{-2}$ & $245 \mathrm{mV}$ & $59.2 \mathrm{mV} \mathrm{dec}-1$ & $1.0 \mathrm{M} \mathrm{KOH}$ & 11 \\
\hline $\mathrm{Co}_{2} \mathrm{P}$ & $10 \mathrm{~mA} \mathrm{~cm}^{-2}$ & $280 \mathrm{mV}$ & $60.4 \mathrm{mV} \mathrm{dec}-1$ & $1.0 \mathrm{M} \mathrm{KOH}$ & 12 \\
\hline $\mathrm{CoP}-\mathrm{N}-\mathrm{C} / \mathrm{CNT}$ & $10 \mathrm{~mA} \mathrm{~cm}^{-2}$ & $270 \mathrm{mV}$ & $56 \mathrm{mV} \mathrm{dec}^{-1}$ & $1.0 \mathrm{M} \mathrm{KOH}$ & 13 \\
\hline $\mathrm{PdP}_{2} /$ Carbon black & $10 \mathrm{~mA} \mathrm{~cm}^{-2}$ & $270 \mathrm{mV}$ & $29.5 \mathrm{mV} \mathrm{dec}-1$ & $1.0 \mathrm{M} \mathrm{KOH}$ & 14 \\
\hline $\mathrm{Co}_{1.5} \mathrm{Fe}_{0.5} \mathrm{P}$ & $10 \mathrm{~mA} \mathrm{~cm}^{-2}$ & $278 \mathrm{mV}$ & $57 \mathrm{mV} \mathrm{dec}^{-1}$ & $1.0 \mathrm{M} \mathrm{KOH}$ & 15 \\
\hline Fe-Co-P nanoboxes & $10 \mathrm{~mA} \mathrm{~cm}^{-2}$ & $269 \mathrm{mV}$ & $31 \mathrm{mV} \mathrm{dec}^{-1}$ & $1.0 \mathrm{M} \mathrm{KOH}$ & 16 \\
\hline NiCoP-O/N-doped C & $10 \mathrm{~mA} \mathrm{~cm}^{-2}$ & $300 \mathrm{mV}$ & $94 \mathrm{mV} \mathrm{dec}^{-1}$ & $1.0 \mathrm{M} \mathrm{KOH}$ & 17 \\
\hline
\end{tabular}



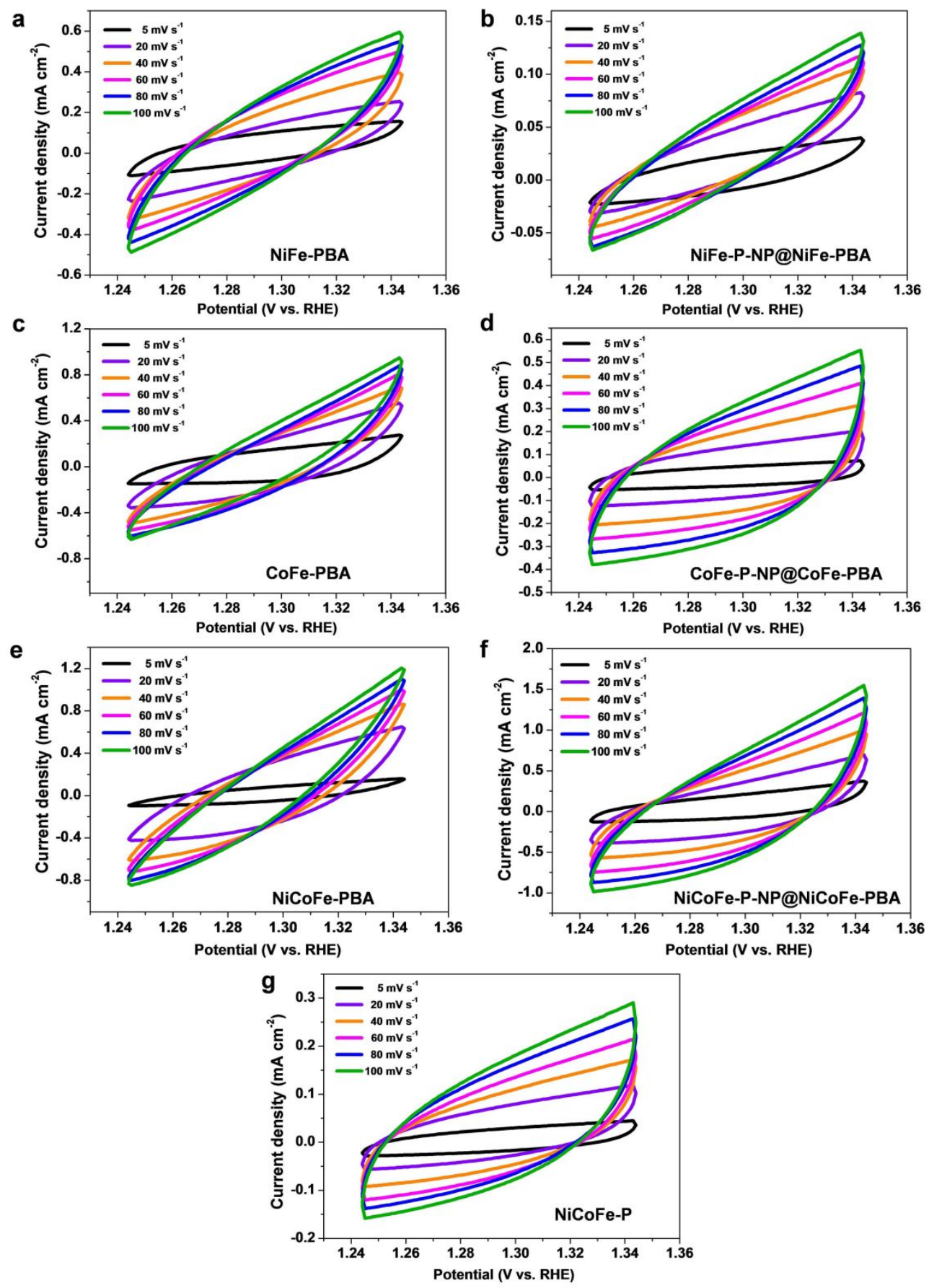

Figure S14. Cyclic voltammograms of (a) NiFe-PBA, (b) NiFe-P-NP@NiFe-PBA, (c) CoFe-PBA, (d) CoFe-P-NP@CoFe-PBA, (e) NiCoFe-PBA, (f) porous NiCoFe-PNP@NiCoFe-PBA nanocage and (g) NiCoFe-P with various scan rates in $1.0 \mathrm{M} \mathrm{KOH}$ solution. 


\section{Electrochemical active surface area (ECSA)}

The electrochemical double-layer capacitance $\left(\mathrm{C}_{\mathrm{dl}}\right)$ was evaluated in a non-faradaic capacitive current range by operating cyclic voltammograms (CVs) to estimate the electrochemical active surface area (ECSA). The ECSA is usually positive correlation to the $\mathrm{C}_{\mathrm{dl}}$ of the electrochemical catalysts. Specifically, CVs with different scan rates are demonstrated in a potential range from 1.244 to $1.344 \mathrm{~V}$ versus the reversible hydrogen electrode (RHE) without redox processes to gain the capacitive current related with the double-layer charging for the OER catalysts. Then, $\mathrm{C}_{\mathrm{dl}}$ can be achieved via plotting the current density difference between the anode and cathode $\left(\Delta j=j_{\mathrm{a}}-j_{\mathrm{b}}\right)$ at $1.29 \mathrm{~V}$ vs. RHE against the different scan rates, and the $\mathrm{C}_{\mathrm{dl}}$ is half of the linear slope, as shown in Figure 4h. The calculated $\mathrm{C}_{\mathrm{dl}}$ values of the NiCoFe-P-NP@NiCoFe-PBA nanocages, NiCoFe-PBA nanocubes, NiCoFe-P nanocages, NiFe-P-NP@NiFe-PBA nanocubes, NiFe-PBA nanocubes, CoFe-P-NP@CoFe-PBA nanoboxes, and CoFePBA nanocubes are shown in the following table (Table S2).

Table S2. The calculated electrochemical double-layer capacitance values of different catalysts.

\begin{tabular}{cc}
\hline Electrochemically active surface areas (ECSAs) & $\propto \mathbf{C}_{\mathbf{d l}}$ values \\
\hline Materials & $\mathbf{C}_{\mathbf{d l}}\left(\mathbf{m F ~} \mathbf{~ m}^{-2}\right)$ \\
\hline NiCoFe-P-NP@NiCoFe-PBA nanocages & 4.93 \\
NiCoFe-PBA nanocubes & 1.69 \\
NiCoFe-P nanocages & 1.05 \\
NiFe-P-NP@NiFe-PBA nanocubes & 0.19 \\
NiFe-PBA nanocubes & 1.51 \\
CoFe-P-NP@CoFe-PBA nanoboxes & 2.42 \\
CoFe-PBA nanocubes & 1.02 \\
\hline
\end{tabular}




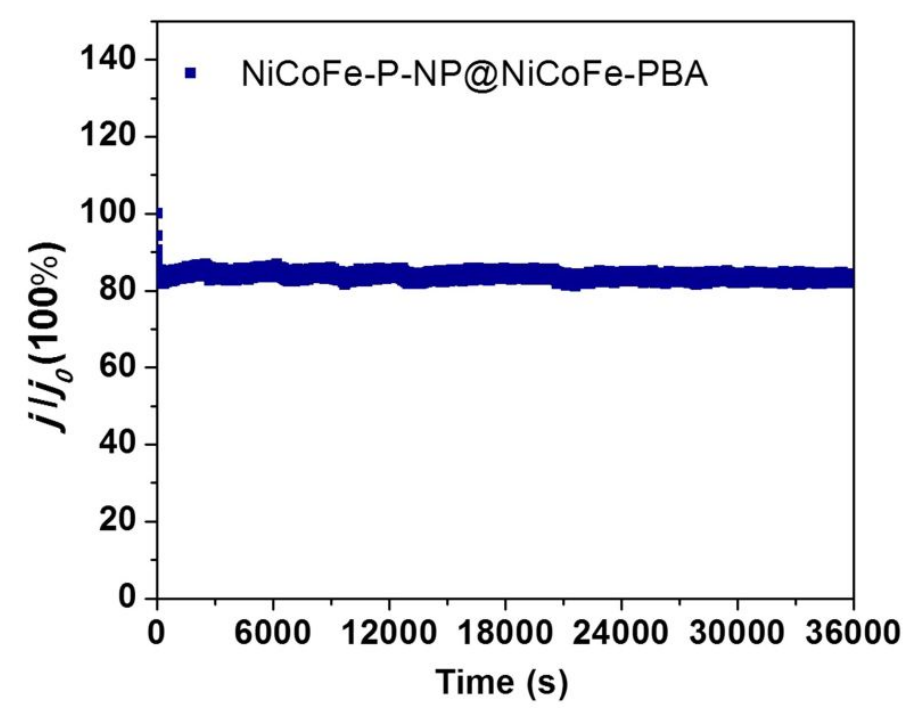

Figure S15. Chronoamperometric responses (i-t) of the porous NiCoFe-PNP@NiCoFe-PBA nanocage catalysts over $36000 \mathrm{~s}$ in $1.0 \mathrm{M} \mathrm{KOH}$.

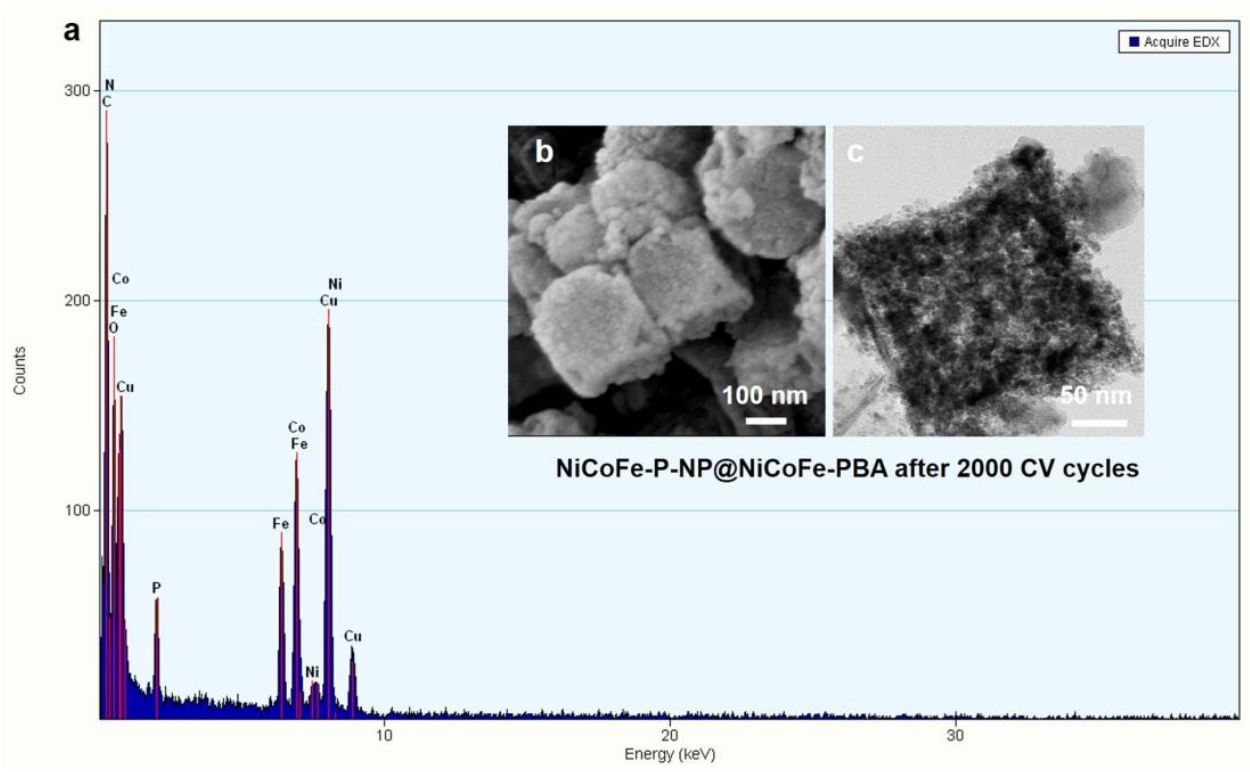

Figure S16. (a) EDX spectrum, (b) SEM and (c) TEM images of the porous NiCoFeP-NP@NiCoFe-PBA nanocages after the 2000 cycles of continuous CV measurements in $1.0 \mathrm{M} \mathrm{KOH}$. 

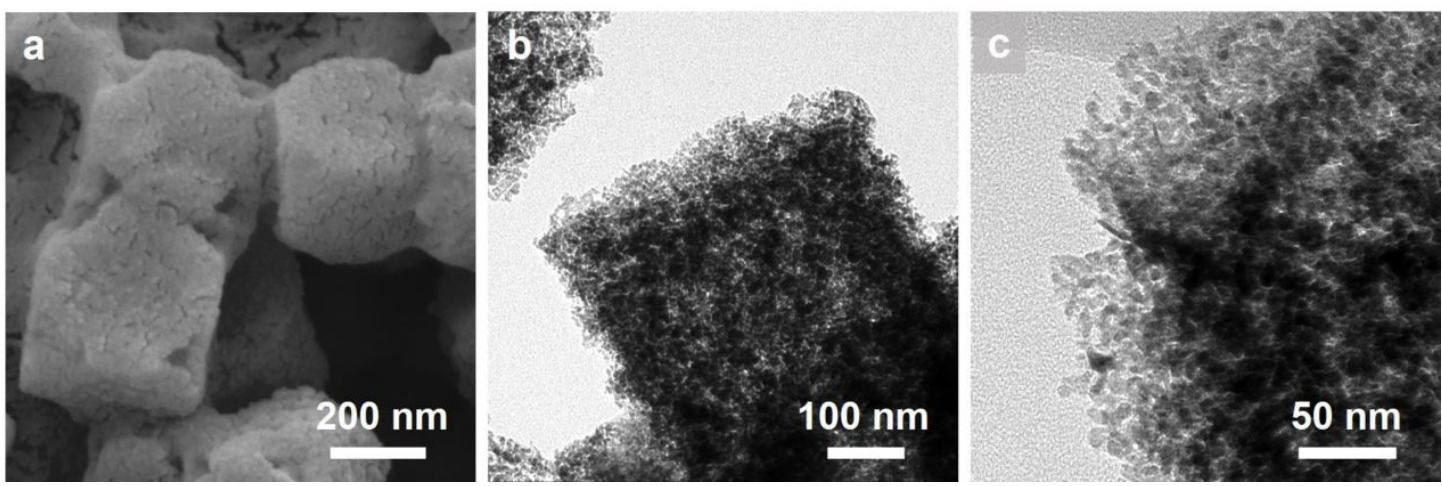

Figure S17. (a) SEM image, (b) and (c) TEM images at different magnifications of the porous NiCoFe-P-NP@NiCoFe-PBA nanocages after the catalytic reaction of oxygen evolution.
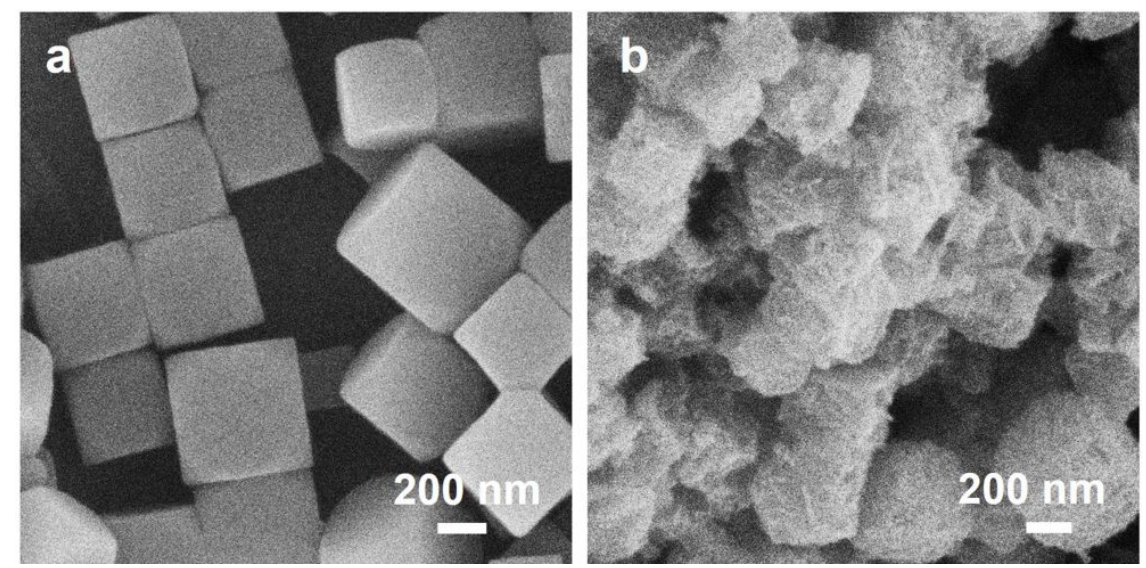

Figure S18. SEM images of the NiCoFe-PBA precursor (a) before and (b) after continuous $\mathrm{CV}$ measurements in $1.0 \mathrm{M} \mathrm{KOH}$. 
Porous NiCoFe-P-NP@NiCoFe-PBA nanocages:

\begin{tabular}{|c|c|c|c|c|c|}
\hline Element & Weight $\mathscr{x}$ & Atomic $\%$ & Uncert. $\%$ & Correction & k-Factor \\
\hline$C(\mathrm{~K})$ & 41.41 & 63.00 & 1.19 & 0.26 & 4. 032 \\
\hline $\mathrm{N}(\mathrm{K})$ & 3.92 & 5.12 & 0.39 & 0.26 & 3.903 \\
\hline$O(\mathrm{~K})$ & 14. 46 & 16.52 & 0.51 & 0.49 & 2. 008 \\
\hline $\mathrm{P}(\mathrm{K})$ & 9.55 & 5.63 & 0.28 & 0.90 & 1.065 \\
\hline $\mathrm{Fe}(\mathrm{K})$ & 12. 24 & 4.00 & 0.35 & 0.99 & 1. 359 \\
\hline $\mathrm{Co}(\mathrm{K})$ & 16.08 & 4. 98 & 0.42 & 0.99 & 1. 444 \\
\hline $\mathrm{Ni}(\mathrm{K})$ & 2. 30 & 0.71 & 0.17 & 0.99 & 1.456 \\
\hline
\end{tabular}

Porous NiCoFe-P-NP@NiCoFe-PBA nanocages after cycles:

\begin{tabular}{|c|c|c|c|c|c|}
\hline Element & Weight $/ 8$ & Atomic $\%$ & Uncert. " o & $\begin{array}{l}\text { Detector } \\
\text { Correction }\end{array}$ & $k-F$ actor \\
\hline $\mathrm{C}(\mathrm{K})$ & 49. 32 & 69.03 & 1.15 & 0.26 & 4. 032 \\
\hline $\mathrm{N}(\mathrm{K})$ & $3.90 \mathrm{I}$ & 4.68 & 0.36 & 0.26 & 3.903 \\
\hline $0(\mathrm{~K})$ & 15.70 & 16.50 & 0.47 & 0.49 & 2.008 \\
\hline $\mathrm{P}(\mathrm{K})$ & 2. 91 & 1.58 & 0.14 & 0.90 & 1.065 \\
\hline $\mathrm{Fe}(\mathrm{K})$ & 10.06 & 3.03 & 0.29 & 0.99 & 1.359 \\
\hline $\mathrm{Co}(\mathrm{K})$ & 16.04 & 4.57 & 0.36 & 0.99 & 1. 444 \\
\hline $\mathrm{Ni}(\mathrm{K})$ & 2.02 & 0.57 & 0.14 & 0.99 & 1. 456 \\
\hline
\end{tabular}

Figure S19. Weight percentages of different elements for the porous NiCoFe-PNP@NiCoFe-PBA nanocages before and after continuous CV measurements (2000 cycles) in $1.0 \mathrm{M} \mathrm{KOH}$.

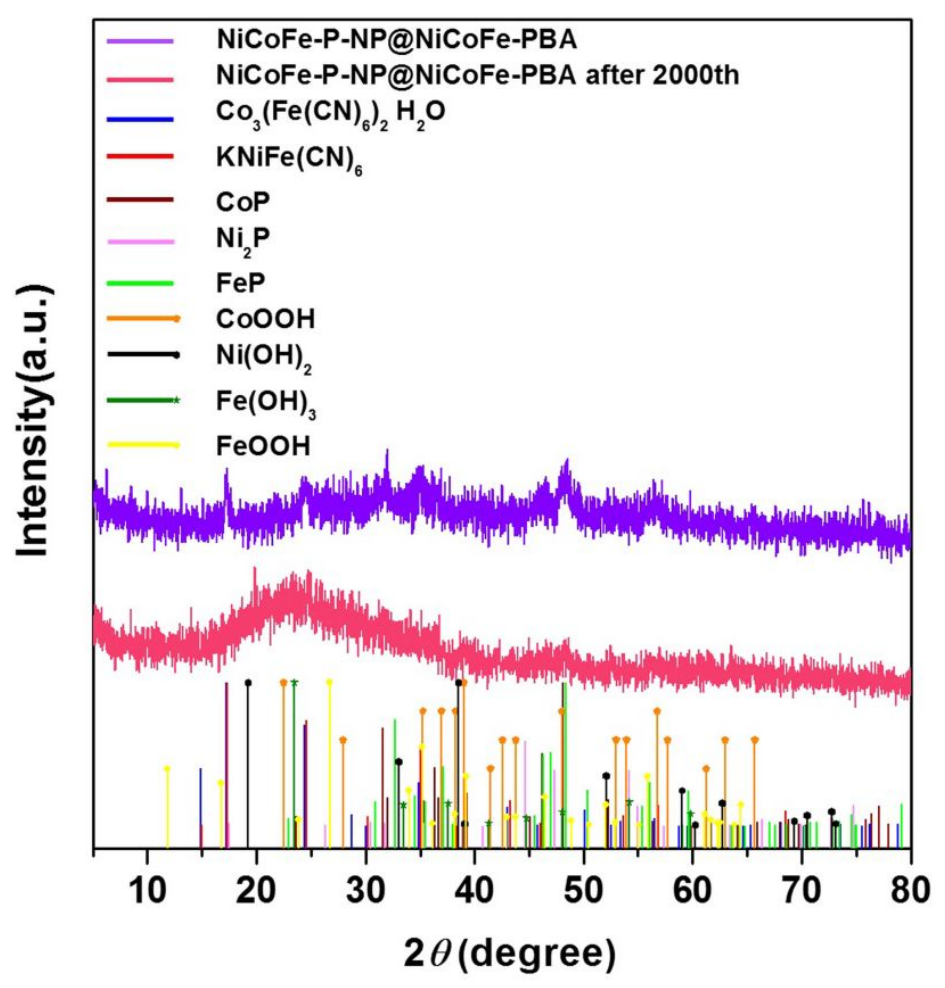

Figure S20. XRD patterns of porous NiCoFe-P-NP@NiCoFe-PBA nanocages before and after continuous CV measurements (2000 cycles) in $1.0 \mathrm{M} \mathrm{KOH}$. 


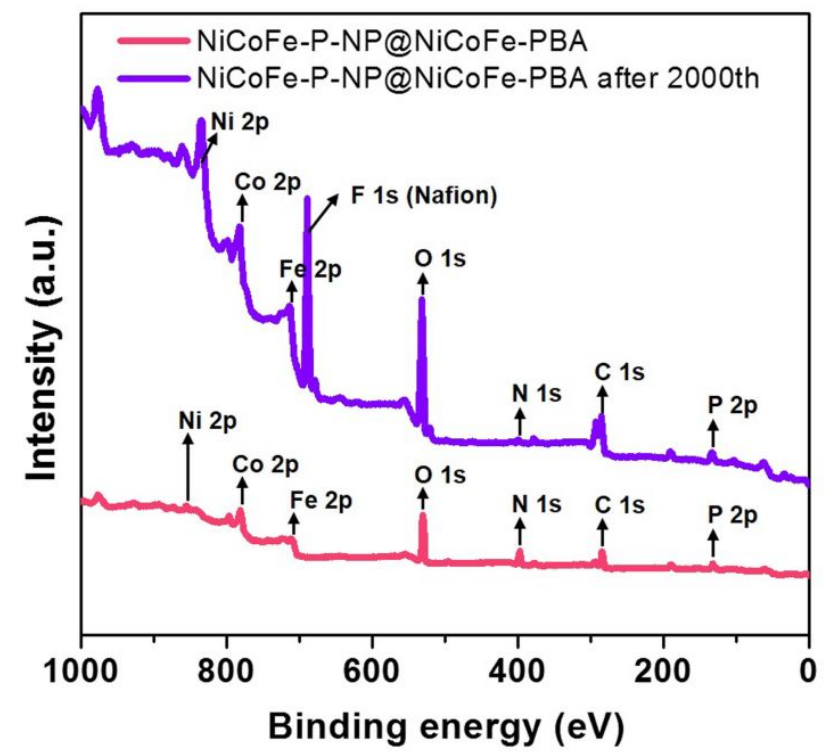

Figure S21. XPS patterns of porous NiCoFe-P-NP@NiCoFe-PBA nanocages before and after continuous CV measurements (2000 cycles) in $1.0 \mathrm{M} \mathrm{KOH}$.

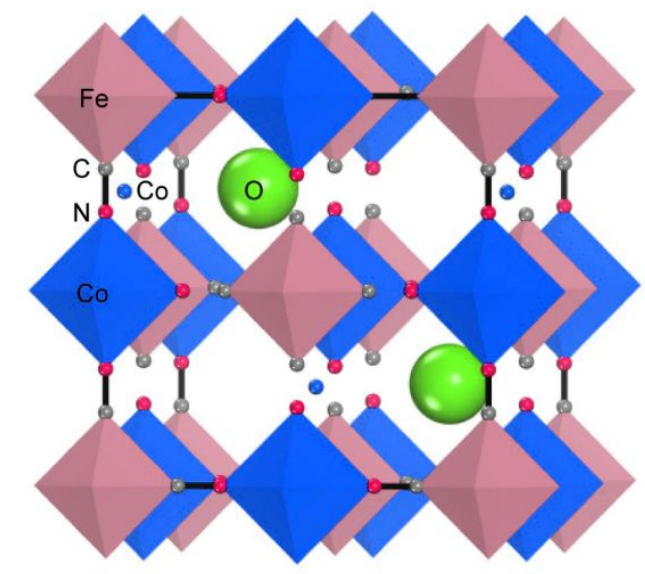

Figure S22. The schematic diagram of $\mathrm{Co}_{3}\left[\mathrm{Fe}(\mathrm{CN})_{6}\right]_{2} \cdot \mathrm{H}_{2} \mathrm{O}$ model. 


\section{REFERENCES}

(1) Popczun, E. J.; Roske, C. W.; Read, C. G.; Crompton, J. C.; McEnaney, J. M.;

Callejas, J. F.; Lewis, N. S.; Schaak, R. E. Highly branched cobalt phosphide nanostructures for hydrogen-evolution electrocatalysis. J. Mater. Chem. A 2015, 3 (10), $5420-5425$.

(2) Hu, G. X.; Tang, Q.; Jiang, D. E. CoP for hydrogen evolution: implications from hydrogen adsorption. Phys. Chem. Chem. Phys. 2016, 18 (34), 23864-23871.

(3) Zhou, G. Y.; Li, M.; Li, Y. L.; Dong, H.; Sun, D. M.; Liu, X.; Xu, L.; Tian, Z. Q.; Tang, Y. W. Regulating the electronic structure of $\mathrm{CoP}$ nanosheets by $\mathrm{O}$ incorporation for high-efficiency electrochemical overall water splitting. Adv. Funct. Mater. 2020, 30 (7), 1905252

(4) Norskov, J. K.; Bligaard, T.; Logadottir, A.; Kitchin, J. R.; Chen, J. G.; Pandelov, S.; Norskov, J. K. Trends in the exchange current for hydrogen evolution. $J$. Electrochem. Soc. 2005, 152 (3), J23-J26.

(5) Peng, J.; Wang, J. S.; Yi, H. C.; Hu, W. J.; Yu, Y. H.; Yin, J. W.; Shen, Y.; Liu, Y.; Luo, J. H.; Xu, Y.; Wei, P.; Li, Y. Y.; Jin, Y.; Ding, Y.; Miao, L.; Jiang, J. J.; Han, J. T.; Huang, Y. H. A dual-insertion type sodium-ion full cell based on high-quality ternary-metal prussian blue analogs. Adv. Energy Mater. 2018, 8 (11), 9.

(6) Wang, J.; Liu, D.; Huang, H.; Yang, N.; Yu, B.; Wen, M.; Wang, X.; Chu, P. K.; Yu, X. F. In-plane black phosphorus/dicobalt phosphide heterostructure for efficient electrocatalysis. Angew. Chem. Int. Ed. 2018, 57 (10), 2600-2604. 
(7) Ji, L.; Wang, J.; Teng, X.; Meyer, T. J.; Chen, Z. CoP nanoframes as bifunctional electrocatalysts for efficient overall water splitting. ACS Catal. 2020, 10 (1), 412-419.

(8) Liu, K.; Zhang, C.; Sun, Y.; Zhang, G.; Shen, X.; Zou, F.; Zhang, H.; Wu, Z.; Wegener, E. C.; Taubert, C. J.; Miller, J. T.; Peng, Z.; Zhu, Y. High-performance transition metal phosphide alloy catalyst for oxygen evolution reaction. ACS nano $\mathbf{2 0 1 8 ,}$ $12(1), 158-167$.

(9) Qiu, B.; Cai, L.; Wang, Y.; Lin, Z.; Zuo, Y.; Wang, M.; Chai, Y. Fabrication of nickel-cobalt bimetal phosphide nanocages for enhanced oxygen evolution catalysis. Adv. Funct. Mater. 2018, 28 (17), 1706008.

(10) Li, G.; Yang, Q.; Rao, J.; Fu, C.; Liou, S. C.; Auffermann, G.; Sun, Y.; Felser, C. In situ induction of strain in iron phosphide $\left(\mathrm{FeP}_{2}\right)$ catalyst for enhanced hydroxide adsorption and water oxidation. Adv. Funct. Mater. 2020, 30 (12), 1907791.

(11) Lv, X.; Li, X.; Yang, C.; Ding, X.; Zhang, Y.; Zheng, Y. Z.; Li, S.; Sun, X.; Tao, X. Large-size, porous, ultrathin NiCoP nanosheets for efficient electro/photocatalytic water splitting. Adv. Funct. Mater. 2020, 30 (16), 1910830.

(12) Li, H.; Li, Q.; Wen, P.; Williams, T. B.; Adhikari, S.; Dun, C.; Lu, C.; Itanze, D.; Jiang, L.; Carroll, D. L.; Donati, G. L.; Lundin, P. M.; Qiu, Y.; Geyer, S. M. Colloidal cobalt phosphide nanocrystals as trifunctional electrocatalysts for overall water splitting powered by a zinc-air battery. Adv. Mater. 2018, 30 (9), 1705796.

(13) Chai, L.; Hu, Z.; Wang, X.; Xu, Y.; Zhang, L.; Li, T. T.; Hu, Y.; Qian, J.; Huang, S. Stringing bimetallic metal-organic framework-derived cobalt phosphide composite for high-efficiency overall water splitting. Adv. Sci. 2020, 7 (5), 1903195. 
(14) Luo, F.; Zhang, Q.; Yu, X.; Xiao, S.; Ling, Y.; Hu, H.; Guo, L.; Yang, Z.; Huang, L.; Cai, W.; Cheng, H. Palladium phosphide as a stable and efficient electrocatalyst for overall water splitting. Angew. Chem. Int. Ed. 2018, 57 (45), 14862-14867.

(15) Yang, S.; Chen, G.; Ricciardulli, A. G.; Zhang, P.; Zhang, Z.; Shi, H.; Ma, J.; Zhang, J.; Blom, P. W. M.; Feng, X. Topochemical synthesis of two-dimensional transition-metal phosphides using phosphorene templates. Angew. Chem. Int. Ed. 2020, $59(1), 465-470$.

(16) Zhang, H.; Zhou, W.; Dong, J.; Lu, X. F.; Lou, X. W. Intramolecular electronic coupling in porous iron cobalt (oxy)phosphide nanoboxes enhances the electrocatalytic activity for oxygen evolution. Energy Environ. Sci. 2019, 12 (11), 3348-3355.

(17) Wang, C.; Chen, W.; Yuan, D.; Qian, S.; Cai, D.; Jiang, J.; Zhang, S. Tailoring the nanostructure and electronic configuration of metal phosphides for efficient electrocatalytic oxygen evolution reactions. Nano Energy 2020, 69, 104453. 